\title{
DNA methylation and expression of homeobox gene family as diagnostic and prognostic markers in human hepatocellular carcinoma
}

\author{
Sumadi Lukman Anwar ${ }^{1}$, Ulrich Lehmann ${ }^{2}$ \\ ${ }^{1}$ Division of Surgical Oncology, Department of Surgery, Faculty of Medicine, \\ Universitas Gadjah Mada, Yogyakarta, Indonesia. 'Institute of Pathology, \\ Medizinische Hochschule Hannover, Hannover, Germany
}

DOI: http://dx.doi.org/10.19106/JMedScieSup004804201632

\section{ABSTRACT}

Homeobox genes consist of a family of evolutionarily-conserved genes that play important roles in morphogenesis, embryogenesis, and determination of cell's fate. Re-expression of embryogenic genes has been associated with carcinogenesis of human cancers. Aberrant expression of homeobox genes has been increasingly found to modulate diverse processes such as cell proliferation, cell death, metastasis, angiogenesis, and DNA repair. We studied DNA methylation and expression of homeobox gene family in human hepatocellular carcinoma (HCC), the fifth most common cancer and the third leading cause of cancer mortality worldwide. We performed microarray for comprehensive DNA methylation and gene expression using primary HCC samples and healthy liver tissues. Confirmation using pyrosequencing and RT-PCR was then performed, followed by clustering both the unsupervised and supervised methods using Qlucore software. Enrichment of homeobox genes both for DNA methylation and gene expression could differentiate $\mathrm{HCC}$ and the healthy liver tissues. Profile of homeobox gene methylation could further predict clinical outcome. Our study revealed an inverse correlation between DNA methylation and gene expression (HOXA9, Spearman $r=-0.49, p=0.002$ ), as well as the correlation between gain of DNA methylation in HOXA9, HOXA13, and MEOX1 and shorter HCC survival (log-rank Mantel-Cox test $p=0.02$, median survival of 50 and 490 weeks, respectively). In conclusion, this study demonstrated the potential roles of DNA methylation and gene expression profiles of homeobox gene family as diagnostic and prognostic marker in patients with HCC.

Keywords: homeobox; DNA methylation; expression; hepatocellular carcinoma; survival 